\title{
Factores motivacionales extrínsecos e intrínsecos en el aula de inglés: análisis empírico ${ }^{1}$
}

\author{
María Elena García Sánchez \\ María Luisa Cruz VARgas \\ Universidad de Almería
}

Recibido: 6 marzo 2012 / Aceptado: 6 septiembre 2012

ISNN: $1697-7467$

\begin{abstract}
RESUMEN: Los factores motivacionales que influyen en el aprendizaje de una lengua extranjera son aspectos de una importancia crucial en el proceso de enseñanza / aprendizaje de un idioma. El objetivo de este artículo es relacionar los factores que describen la personalidad del alumno con los componentes motivacionales externos en el aula. Más concretamente, en este artículo presentamos un estudio empírico de la frecuencia con que ocurren los factores motivacionales externos, lo que nos ayudará a crear un entorno agradable y propicio en el aula de una lengua extranjera. En particular, llevaremos a cabo un análisis factorial para agrupar los diferentes factores motivacionales externos en perfiles y comprobar cuáles son más utilizados y cuáles están siendo infrautilizados.

Palabras clave: Motivación, factor, extrínseco, proceso de enseñanza, análisis de componentes principales.
\end{abstract}

Extrinsic and Intrinsic Motivational Factors in the English Classroom: Empirical Analysis

\begin{abstract}
The motivational factors that influence the learning of a foreign language are aspects of a crucial importance in the process of teaching / learning of a language. The aim of this paper is to relate the factors describing the personality of students with the extrinsic motivational components in a classroom. More specifically, in this paper we will present an empirical study of the frequency with which the motivational external factors occur; this help us to create a nice and propitious environment in the classroom of a foreign language. In particular, we will carry out a factorial analysis to group the different motivational external factors into components and to verify what of them are more used and what are underused.
\end{abstract}

Keywords: Motivation, factor, extrinsic, learning process, main factors analysis.

\section{INTRODUCCIÓN}

Recientemente, la importancia de los métodos cualitativos y cuantitativos en la investigación en Lingüística Aplicada ha sido puesta de manifiesto por Dörnyei (2007). Esta obra

\footnotetext{
${ }^{1}$ Los autores agradecen los comentarios y sugerencias de un evaluador anónimo.
} 
muestra una gran variedad de metodologías que pueden ser aplicadas a diversos procedimientos de investigación. En esta línea se sitúa el presente trabajo aplicado a la motivación en un aula de lengua extranjera, donde, en primer lugar, vamos a efectuar un repaso de los tipos de estudios empíricos llevados a cabo sobre el tema de la motivación en el aula.

Como es sabido, en el aprendizaje de una L2 o LE intervienen múltiples factores que pueden agruparse en tres grandes grupos (Ellis, 1994:193): (a) factores externos al individuo: input, relación con el medio, contexto y situación de aprendizaje; (b) factores internos: lengua materna, conocimiento del mundo y conocimiento lingüístico; y (c) factores individuales: edad, motivación, aptitud, etc. En este contexto, la relevancia de esta investigación se justifica por la enorme trascendencia de la variable motivación en el rendimiento escolar del alumnado de lengua extranjera como ponen de manifiesto, entre otros, Pérez Puente (2006), Dörnyei (2008:23), López García (2009:1-9) y Ramos Nieto (2011:39-49). Por este motivo, se lleva a cabo un estudio de la frecuencia de los factores motivacionales externos que los docentes emplean en el aula de lengua extranjera. Resulta de gran interés agrupar los factores motivacionales en componentes principales, para saber qué grupos son los que más se utilizan o, dicho de otro modo, para poner especial énfasis en la implantación en el aula de los menos utilizados.

Clément et al. (1994), citados por Dörnyei (1998:117), proponen un constructo de motivación tripartito entre los estudiantes, consistente en la integración, la autoconfianza lingüística y la valoración del contexto de la clase. Más tarde, Dörnyei (1994), citado por Dörnyei (1998:117), partiendo del trabajo de Clément et al., propone una lista de componentes motivacionales categorizados en tres dimensiones: el nivel del lenguaje, el nivel del estudiante y el nivel de la situación de aprendizaje. A su vez, divide la última dimensión en tres grupos de componentes motivacionales: específicas del curso, específicas del profesor y específicas del grupo. Observemos que tanto los modelos de Clément et al. (1994) y de Dörnyei (1994) incluyen factores motivacionales extrínsecos e intrínsecos, y que no llegan a ellos a través de un estudio empírico.

Por su parte, Barrios Espinosa (1997:17) manifiesta las dificultades en la identificación y medición de las variables que describen los factores afectivos que inciden en la adquisición de una lengua extranjera. En esta línea, Marins de Andrade (2010:141) demuestra con estudiantes de E/LE brasileños que las estrategias afectivas comprenden sentimientos, actitudes y motivaciones, y que los alumnos encuestados resaltan las artes (cine, literatura y música) como muy motivadoras.

Posteriormente, Williams y Burden (1997), citados por Dörnyei (1998:117), proponen un marco teórico en donde enmarcan los factores motivaciones externos en cuatro dimensiones: la naturaleza de la interacción, el contexto del aprendizaje, el contexto amplio y otros factores significativos. En este punto, tenemos que decir que el modelo de Williams y Burden es el que más se aproxima a las conclusiones obtenidas en este trabajo (véase la Sección 3).

Por su parte, Dörnyei y Csizér (1998:203) señalaron la escasa disponibilidad de análisis multivariantes en el marco de esta investigación y llevaron a cabo una encuesta entre profesores y alumnos acerca de la importancia de 51 estrategias motivacionales y su frecuencia en la práctica docente. Mediante un análisis Cluster, estas estrategias se agruparon en 18 macroestrategias de las que los autores han seleccionado las 10 primeras, lo que, entre otras cosas, ponen a la luz las (macro)estrategias que son infrautilizadas en la práctica docente. En este mismo sentido, Bernaus, Wilson y Gardner (2009:25), utilizando el análisis de 
componentes principales, demuestran que la motivación del profesor está relacionada con el uso que éste hace de estrategias motivadoras que, a su vez, se relacionan con la motivación del alumnado y sus logros en inglés. Para ello, estudiaron las siguientes variables: logros en inglés, integración, actitudes hacia la situación de aprendizaje, motivación, preocupación por el lenguaje, orientación instrumental, ánimo de los padres, estrategias tradicionales e innovadoras de los profesores, estrategias tradicionales e innovadoras del alumnado y motivación del profesor.

Liuolienè y Metiūnienė (2006:93) estudian los tres enfoques principales de la psicología motivacional (teoría del valor de la expectativa, teoría dirigida hacia el objetivo y teoría de la autodeterminación) utilizando el método ANOVA, concluyendo que:

- La motivación en el aprendizaje es una fuerza orientada hacia el aprendizaje de una lengua extranjera.

- Los deseos y necesidades del estudiante para trabajar independientemente dependen de su motivación, actitud y responsabilidad.

- Cuanto mayor sea la motivación, mayor aprendizaje autónomo querrán tener los estudiantes.

Estas conclusiones se verán corroboradas en nuestro análisis empírico al final del presente trabajo.

Por último, Gómez Martínez (2008:217) utiliza el análisis factorial para analizar el tipo de orientación (desde instrumental a integradora, pasando por un abanico de tipos de orientaciones que duplican la dicotomía clásica) a una muestra de estudiantes. Su investigación conduce a cuatro perfiles que se pueden entender en un continuo que va desde los dos perfiles clásicos (claramente integrador y claramente instrumental) hasta dos perfiles adicionales, que corresponden a las nuevas contribuciones originales. En el mismo sentido, Uribe, Gutiérrez y Madrid (2008:85) concluyen que el alumnado español parece haber desarrollado actitudes ligeramente positivas que favorecen la aparición de una motivación instrumental cuando aprenden un idioma extranjero. Estas actitudes se ven afectadas por el sexo, la nota media en inglés, las clases particulares, la clase social y el curso de iniciación al inglés. Utilizando análisis de la varianza, los autores no encontraron correlaciones importantes entre actitudes y variables independientes. Únicamente, observaron que las chicas muestran actitudes más positivas que los chicos hacia las actividades de clase y que los alumnos que comienzan a estudiar el inglés antes tienen actitudes más favorables hacia las tareas de clase.

La organización de este artículo es la siguiente: después de una introducción en la que se ponen de manifiesto algunos trabajos que utilizan métodos cuantitativos para el análisis de la motivación en el aula, la Sección 2 fija los objetivos principales y secundarios de este artículo, así como la metodología a emplear, basada en el análisis de componentes principales. La Sección 3 describe el análisis cuantitativo como resultado de las encuestas pasadas a tres colectivos de estudiantes de ESO, $1^{\circ}$ y $2^{\circ}$ de Bachillerato. Por último, la Sección 4 recoge las principales conclusiones del estudio. 


\section{Objetivos Y Metodología del trabajo}

El objetivo principal de este trabajo es doble. Por una parte, se pretende estudiar los factores de la personalidad que están relacionados con los componentes motivacionales en el aula, y, por la otra, conocer los diferentes niveles de motivación extrínseca en el alumnado de la asignatura de inglés.

Por su parte, los objetivos secundarios son:

- Conocer el estado inicial de motivación del alumnado de lengua inglesa.

- Comprobar la influencia de la motivación extrínseca en el proceso de aprendizaje.

- Estudiar el efecto motivador de las situaciones que se presentan en la dinámica del aula: felicitaciones y reproches, dificultad de las tareas, éxitos y fracasos, negociación curricular, participación en clase, etc.

- Conocer la frecuencia en el uso de los factores motivadores extrínsecos en el proceso de enseñanza y aprendizaje.

- Conocer el efecto motivador de las tareas y ejercicios de clase.

- Agrupar los diferentes factores motivacionales externos en niveles o dimensiones para simplificar la adopción de estrategias docentes por parte del profesor.

La metodología a emplear será la siguiente:

1. Técnicas e instrumentos de recogida de datos. Tanto la medición de la variable "personalidad", como las variables "componentes motivacionales", "resultado" y "frecuencia de los factores motivadores externos en el aula" se va a realizar mediante la cumplimentación de un cuestionario (Madrid, 1999). Los cuestionarios se componen de 13, 5, 1 y 18 preguntas, respectivamente, que van a medir diferentes subvariables. A su vez, cada ítem tiene cinco posibles respuestas de valoración (1, 2, 3,4 y 5). Por tanto, se trata de variables medibles a través de una escala de Likert, que permite medir el sentido y la intensidad de las actitudes, y que es bastante fácil de administrar. No obstante, esta escala adolece de ponderación en las respuestas.

2. Descripción de la muestra. La muestra empleada para el primer análisis ha estado formada por 47 alumnos y 39 alumnas de $3^{\circ}$ de ESO y de $1^{\circ}$ de Bachillerato del Instituto de Enseñanza Secundaria "Bahía de Almería". El segundo análisis ha considerado la clase de inglés de $1^{\circ}$ y $2^{\circ}$ de Bachillerato del Instituto de Enseñanza Secundaria "Alhamilla" de Almería, durante el curso académico 2010/2011. Este colectivo estaba formado por 13 chicas y 17 chicos. Ambos análisis recogen la opinión de alumnos procedentes de distintos centros escolares y de diversa extracción social, teniendo en cuenta que el primero se encuentra en el extrarradio de Almería y que el segundo se encuentra situado en la zona centro.

3. Breve descripción del profesorado y de los medios a su disposición. De acuerdo con la información facilitada por los propios docentes, el profesorado involucrado en esta investigación cuenta con entre 10 y 25 años de experiencia docente. Los centros encuestados carecen de lectores nativos de apoyo para las clases de inglés. En cuanto al material utilizado, podemos destacar el uso del libro de texto, audiciones con $\mathrm{CD}$, fotocopias adicionales para las actividades de refuerzo, visionado de películas, 
libros de lectura y realización de viajes con los alumnos. El profesorado asiste regularmente a cursos de formación de didáctica del inglés y de nuevas tecnologías, tanto en el Centro de Profesores (CEP) como en otros centros de Secundaria.

4. Técnicas para el análisis de datos. En nuestro trabajo, utilizaremos el análisis multivariante, más concretamente, el análisis factorial. De acuerdo con Madrid (2001), esta técnica examina la interrelación entre variables para descubrir cuántos factores independientes se pueden identificar en los datos y nos explican una proporción de la varianza de los sujetos. Para una descripción más detallada de este método de investigación, puede consultarse a Dörnyei (2007).

\section{Resultados}

Siguiendo a Madrid (1999), hemos pasado un cuestionario acerca de personalidad, motivación y rendimiento en el aula de inglés. Las respuestas posibles (variables para el análisis) han sido:

- V1: Soy tímido.

- V2: Soy extrovertido.

- V3: Soy autoritario.

- V4: Mi cultura es superior.

- V5: Me gusta intervenir en los asuntos de los demás.

- V6: No me convence vivir en España.

- V7: Soy constante.

- V8: Me gusta participar.

- V9: Me identifico con las demás personas.

- V10: Soy tolerante.

- V11: Tengo aguante en situaciones de desorientación.

- V12: Siento ansiedad.

- V13: El idioma extranjero me interesa, me gusta y me esfuerzo.

- V14: Grado de interés.

- V15: Grado de atención.

- V16: Esfuerzo.

- V17: Grado de dedicación y constancia.

- V18: Grado de satisfacción global.

- V19: Nota media.

Con las respuestas obtenidas, hemos llevado a cabo un análisis factorial cuyas conclusiones iremos comentando a continuación. Si el lector está interesado en profundizar en esta técnica del análisis multivariante, puede consultar el manual de Hair, Anderson, Tatham y Black (2007) o alguna aplicación empírica en Barrera y Reyes (2003:285). En primer lugar, la tabla 1 muestra las medias y las desviaciones típicas de las respuestas obtenidas en cada uno de los ítems del cuestionario, con 86 respuestas cada uno de ellos: 
Tabla 1. Medias y desviaciones típicas de cada item.

\begin{tabular}{|c|c|c|c|}
\hline \multicolumn{4}{|c|}{ Estadísticos descriptivos } \\
\hline Variable & Media & Desviación típica & N. del análisis \\
\hline V1 & 2,5698 & 1,04646 & 86 \\
\hline V2 & 3,3372 & 1,06947 & 86 \\
\hline V3 & 2,5581 & 1,20396 & 86 \\
\hline V4 & 3,0116 & 1,41832 & 86 \\
\hline V5 & 1,7674 & 1,12380 & 86 \\
\hline V6 & 1,8837 & 1,17241 & 86 \\
\hline V7 & 3,6279 & 1,09594 & 86 \\
\hline V8 & 3,1977 & 1,15626 & 86 \\
\hline V9 & 3,2558 & 1,12914 & 86 \\
\hline V10 & 3,5814 & 1,12161 & 86 \\
\hline V11 & 3,0465 & 1,07273 & 86 \\
\hline V12 & 1,8721 & 1,24425 & 86 \\
\hline V13 & 3,7326 & 1,06743 & 86 \\
\hline V14 & 3,6977 & 0,84114 & 86 \\
\hline V15 & 3,6279 & 0,94616 & 86 \\
\hline V16 & 3,7674 & 0,87682 & 86 \\
\hline V17 & 3,3721 & 0,94616 & 86 \\
\hline V18 & 3,4419 & 0,91529 & 86 \\
\hline V19 & 3,2791 & 0,97801 & 86 \\
\hline
\end{tabular}

La tabla 2 muestra la varianza total explicada (en \%) y la varianza acumulada por cada una de las posibles componentes del análisis de componentes principales: 
Tabla 2. Varianza total explicada y acumulada por cada item.

\begin{tabular}{|c|c|c|c|}
\hline \multicolumn{4}{|c|}{ Autovalores iniciales } \\
\hline Componente & Total & \% de la varianza & \% acumulado \\
\hline V1 & 4,185 & 22,029 & 22,029 \\
\hline V2 & 2,547 & 13,405 & 35,434 \\
\hline V3 & 1,631 & 8,585 & 44,019 \\
\hline V4 & 1,517 & 7,986 & 52,005 \\
\hline V5 & 1,266 & 6,663 & 58,668 \\
\hline V6 & 1,139 & 5,994 & 64,663 \\
\hline V7 & 1,022 & 5,378 & 70,041 \\
\hline V8 & 0,897 & 4,719 & 74,760 \\
\hline V9 & 0,711 & 3,744 & 78,505 \\
\hline V10 & 0,612 & 3,220 & 81,725 \\
\hline V11 & 0,537 & 2,826 & 84,550 \\
\hline V12 & 0,523 & 2,752 & 87,303 \\
\hline V13 & 0,490 & 2,577 & 89,879 \\
\hline V14 & 0,441 & 2,322 & 92,201 \\
\hline V15 & 0,418 & 2,200 & 94,401 \\
\hline V16 & 0,339 & 1,783 & 96,184 \\
\hline V17 & 0,280 & 1,473 & 97,657 \\
\hline V18 & 0,241 & 1,266 & 98,923 \\
\hline V19 & 0,205 & 1,077 & 100,000 \\
\hline
\end{tabular}

Teniendo en cuenta los porcentajes de varianza explicada, podríamos agrupar las 19 variables en 7 factores $(70,041 \%$ de varianza explicada), por lo que hemos procedido a llevar a cabo un análisis factorial forzado a 7 factores.

El gráfico de sedimentación (figura 3) ofrece los autovalores correspondientes a cada una de las variables: 


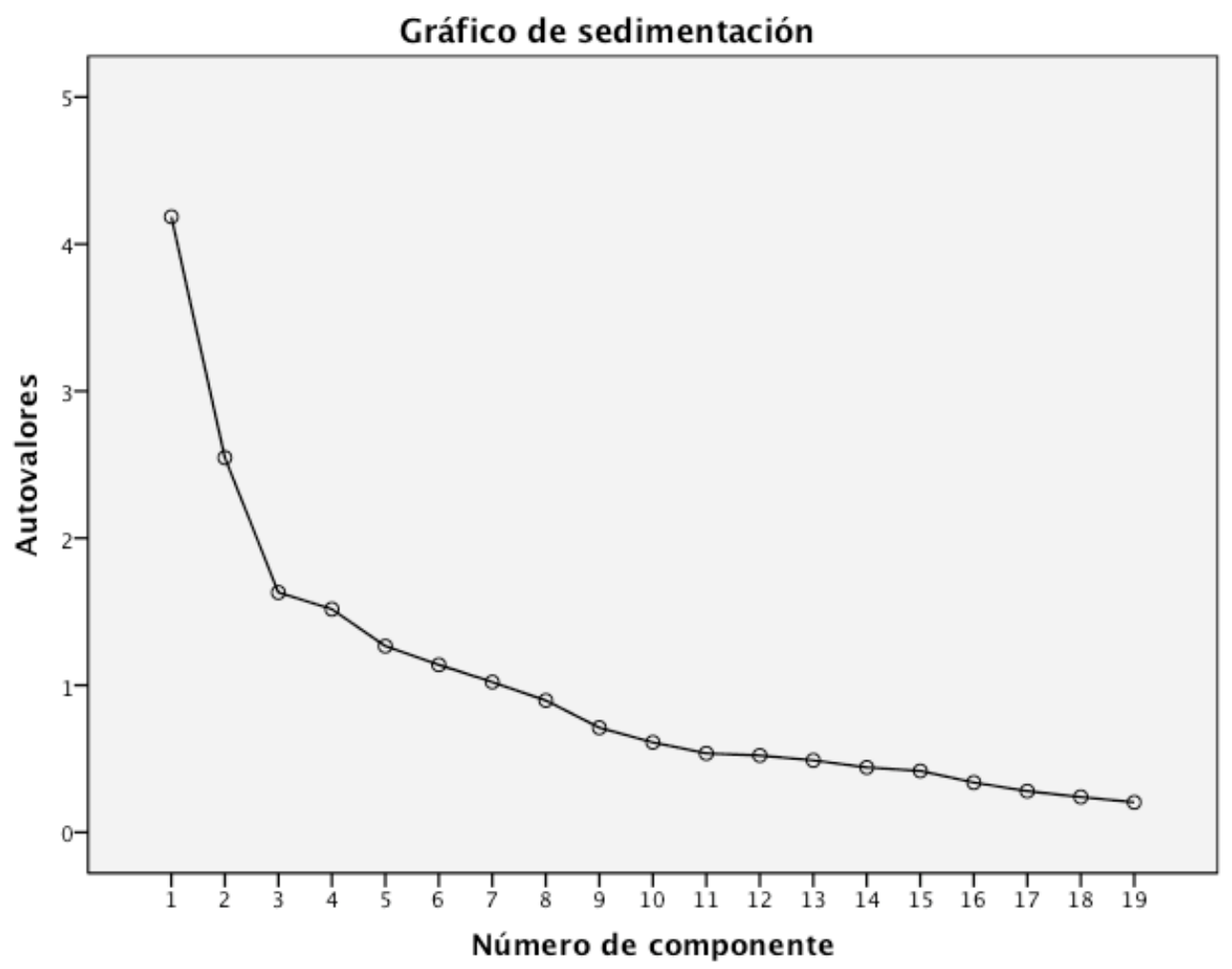

Figura 1. Gráfico de sedimentación de las variables en estudio. 
La matriz de componentes rodados arroja los siguientes resultados:

Tabla 3. Matriz de componentes rodados.

\begin{tabular}{|c|c|c|c|c|c|c|c|}
\hline \multicolumn{8}{|c|}{ Matriz de componentes rotados } \\
\hline \multirow{2}{*}{ Variable } & \multicolumn{7}{|c|}{ Componente } \\
\hline & 1 & 2 & 3 & 4 & 5 & 6 & 7 \\
\hline V1 & 0,221 & $-0,645$ & 0,228 & $-0,230$ & 0,106 & 0,235 & 0,089 \\
\hline $\mathrm{V} 2$ & $-0,079$ & 0,762 & 0,153 & $-0,078$ & $-0,138$ & $-0,299$ & 0,163 \\
\hline V3 & 0,092 & 0,066 & 0,046 & 0,830 & 0,170 & $-0,062$ & $-0,027$ \\
\hline V4 & 0,035 & 0,063 & $-0,285$ & $-0,056$ & $-0,690$ & 0,179 & $-0,115$ \\
\hline V5 & $-0,194$ & 0,131 & 0,044 & 0,754 & $-0,278$ & 0,004 & 0,157 \\
\hline V6 & $-0,033$ & $-0,041$ & 0,797 & 0,075 & 0,070 & 0,225 & $-0,021$ \\
\hline V7 & 0,489 & 0,083 & 0,603 & $-0,055$ & $-0,015$ & $-0,050$ & 0,128 \\
\hline V8 & 0,298 & 0,787 & 0,124 & 0,011 & 0,167 & $-0,003$ & $-0,055$ \\
\hline V9 & $-0,042$ & 0,722 & $-0,067$ & 0,148 & 0,171 & 0,223 & 0,003 \\
\hline V10 & 0,271 & 0,245 & $-0,020$ & $-0,119$ & 0,720 & 0,190 & 0,124 \\
\hline V11 & $-0,034$ & 0,015 & $-0,091$ & 0,093 & 0,196 & 0,061 & 0,892 \\
\hline V12 & $-0,011$ & $-0,160$ & 0,169 & $-0,236$ & $-0,243$ & 0,723 & 0,150 \\
\hline V13 & 0,201 & 0,051 & 0,710 & 0,050 & 0,218 & $-0,266$ & $-0,255$ \\
\hline V14 & 0,737 & 0,091 & 0,071 & 0,036 & $-0,018$ & 0,079 & 0,233 \\
\hline V15 & 0,680 & $-0,113$ & 0,263 & $-0,102$ & $-0,064$ & $-0,246$ & 0,211 \\
\hline V17 & 0,760 & $-0,074$ & 0,215 & 0,011 & 0,150 & $-0,119$ & $-0,278$ \\
\hline V17 & 0,830 & 0,009 & 0,000 & 0,042 & 0,147 & $-0,213$ & $-0,099$ \\
\hline V18 & 0,794 & 0,005 & $-0,055$ & $-0,107$ & 0,055 & 0,099 & $-0,157$ \\
\hline V19 & 0,351 & 0,008 & 0,140 & $-0,161$ & $-0,278$ & $-0,613$ & 0,056 \\
\hline
\end{tabular}


Por tanto, cada una de las variables anteriores puede agruparse en los siguientes componentes o factores:

Tabla 4. Agrupación de las variables por componentes.

\begin{tabular}{|c|c|c|c|}
\hline Factores & Variables & Media & DT \\
\hline Factor 1 & $\begin{array}{l}\text { V14: Grado de interés } \\
\text { V15: Grado de atención } \\
\text { V16: Esfuerzo } \\
\text { V17: Grado de dedicación y constancia } \\
\text { V18: Grado de satisfacción global }\end{array}$ & $\begin{array}{l}3,6977 \\
3,6279 \\
3,7674 \\
3,3721 \\
3,4419\end{array}$ & $\begin{array}{l}0,84114 \\
0,94616 \\
0,87682 \\
0,94616 \\
0,91529\end{array}$ \\
\hline Factor 2 & $\begin{array}{l}\text { V1: Soy tímido } \\
\text { V2: Soy extrovertido } \\
\text { V8: Me gusta participar } \\
\text { V9: Me identifico con las demás personas }\end{array}$ & $\begin{array}{l}2,5698 \\
3,3372 \\
3,1977 \\
3,2558\end{array}$ & $\begin{array}{l}1,04646 \\
1,06947 \\
1,15626 \\
1,12914\end{array}$ \\
\hline Factor 3 & $\begin{array}{l}\text { V6: No me convence vivir en España } \\
\text { V7: Soy constante } \\
\text { V13: El idioma extranjero me interesa, me } \\
\text { gusta y me esfuerzo }\end{array}$ & $\begin{array}{l}1,8837 \\
3,6279 \\
3,7326\end{array}$ & $\begin{array}{l}1,17241 \\
1,09594 \\
1,06743\end{array}$ \\
\hline Factor 4 & $\begin{array}{l}\text { V3: Soy autoritario } \\
\text { V5: Me gusta intervenir en los asuntos de los } \\
\text { demás }\end{array}$ & $\begin{array}{l}2,5581 \\
1,7674\end{array}$ & $\begin{array}{l}1,20396 \\
1,12380\end{array}$ \\
\hline Factor 5 & $\begin{array}{l}\text { V4: Mi cultura es superior } \\
\text { V10: Soy tolerante }\end{array}$ & $\begin{array}{l}3,0116 \\
3,5814\end{array}$ & $\begin{array}{l}1,41832 \\
1,12161\end{array}$ \\
\hline Factor 6 & $\begin{array}{l}\text { V12: Siento ansiedad } \\
\text { V19: Nota media }\end{array}$ & $\begin{array}{l}1,8721 \\
3,6977\end{array}$ & $\begin{array}{l}1,24425 \\
0,84114\end{array}$ \\
\hline Factor 7 & $\begin{array}{l}\text { V11: Tengo aguante en situaciones de deso- } \\
\text { rientación }\end{array}$ & 3,0465 & 1,07273 \\
\hline
\end{tabular}

Las componentes anteriormente obtenidas podrían describirse como sigue:

- Componente 1: Componente motivacional.

- Componente 2: Factor de personalidad de proyección hacia los demás.

- Componente 3: Factor de personalidad de búsqueda de otras culturas.

- Componente 4: Factor de personalidad de implantación de ideas. 
- Componente 5: Factor de autoevaluación de cultura y conocimientos.

- Componente 6: Factor de personalidad de búsqueda de buenas notas.

- Componente 7: Factor de personalidad de resistencia ante situaciones difíciles.

A continuación, pasamos a comentar los resultados obtenidos en el análisis anterior. Comenzando con el componente o factor 1, al que hemos denominado "componente motivacional" y partiendo de las medias obtenidas, cabe destacar que, para la mayoría de los encuestados, todas las variables reciben una valoración altamente positiva, lo que indica que su componente motivacional es elevada con respecto al interés, la atención, el grado de dedicación y constancia, así como su grado de satisfacción global. Como dato a destacar, la media más alta está relacionada con el esfuerzo que los alumnos realizan, lo que nos indica que éstos tienen una elevada percepción de su esfuerzo personal.

Con respecto al factor 2 donde se analiza la componente de la "personalidad de proyección hacia los demás", se observa una diferencia significativa entre las medias de tres de las cuatro variables con la cuarta de ellas, correspondiente al hecho de ser tímido. En general, los alumnos declaran que son extrovertidos, que les gusta participar en clase y que se identifican con las demás personas; y esto queda corroborado por el hecho de tener un bajo grado de timidez, según la media obtenida, lo cual es un dato importante a la hora de aprender una lengua extranjera.

En el siguiente componente, se incluye el "factor de personalidad de búsqueda de otras culturas" y, de las medias obtenidas, se extrae la conclusión de que el idioma extranjero les interesa, les gusta y se esfuerzan por aprenderlo con una media elevada, y a su vez son pocos los que dicen que no les convence vivir en España, lo cual evidencia claramente que, aunque les interese aprender el inglés como lengua extranjera, quieren seguir viviendo en España.

$\mathrm{Al}$ abordar el componente 4 relacionado con el "factor de personalidad de implantación de ideas", nos damos cuenta de que los alumnos manifiestan que no son muy autoritarios y que no les gusta intervenir demasiado en los asuntos de los demás, ya que la media es baja en cuanto a la primera variable y bastante baja en cuanto a la segunda.

Los resultados de las medias obtenidas en torno al componente o factor 5 relacionado con la "autoevaluación de cultura y conocimientos", indican que el alumnado considera, por un lado, que su cultura es superior y, por otro lado, que son tolerantes, lo cual entra un poco en contradicción sin llegar a ser excesivamente llamativo ya que en la variable "mi cultura es superior" se obtiene una media de 3 sobre 5 , que es significativamente más baja que la correspondiente a la variable "soy tolerante" a un nivel del $5 \%$.

Con respecto al factor 6, relacionado con la "búsqueda de buenas notas", son pocos los alumnos que manifiestan sentirse en estado de ansiedad con las calificaciones, y son bastantes los que indican que están contentos con su nota media (alrededor de notable), según los datos obtenidos.

En cuanto al componente 7 que tiene que ver con el "factor de personalidad de resistencia ante situaciones difíciles" tales como situaciones de incomprensión, desorientación, confusión, ambigüedad, frustración y angustia que se producen durante el período de aprendizaje de la lengua extranjera, según la media obtenida, un 3 sobre 5 , se puede observar que los alumnos manifiestan tener un moderado o cierto nivel de resistencia frente a tales situaciones, pero que dicho nivel no es muy alto. 
Finalmente, como podemos comprobar en el análisis anterior, el componente motivacional interno se agrupa en un solo factor en el que no aparece ninguna variable adicional. Por tanto, podemos concluir que la motivación intrínseca se percibe como un concepto único, con distintos aspectos, para cualquier alumno, independientemente de su factor de personalidad. Esto hace que, en el segundo análisis, intentemos agrupar los factores motivacionales extrínsecos para asociarlos con los distintos factores que definen la personalidad.

Nuevamente, siguiendo a Madrid (1999), hemos pasado un cuestionario acerca de la frecuencia con la que se utilizan los factores motivadores externos en el aula de inglés. Las respuestas posibles (variables para el análisis) han sido:

- V1: Felicitación.

- V2: Reproches.

- V3: Adaptación de las tareas por parte del profesor.

- V4: Desafío intelectual.

- V5: Obtención de buenos resultados.

- V6: Trabajo en equipo.

- V7: Negociación de las decisiones curriculares.

- V8: Autoevaluación.

- V9: Trabajo individual.

- V10: Participación.

- V11: Clases en inglés.

- V12: Cobertura de necesidades e intereses.

- V13: Actuar ante audiencia.

- V14: Competir.

- V15: Información sobre contenidos y objetivos.

- V16: No participación en clase.

- V17: Descubrimiento de las cosas.

- V18: Medios audiovisuales.

Con las respuestas obtenidas, hemos llevado a cabo un análisis factorial cuyas conclusiones iremos comentando a continuación. En primer lugar, la tabla 5 muestra las medias y las desviaciones típicas de las respuestas obtenidas en cada uno de los ítems del cuestionario, con 30 respuestas cada uno de ellos: 
Tabla 5. Medias y desviaciones típicas de cada nuevo ítem.

\begin{tabular}{|c|c|c|c|}
\hline \multicolumn{4}{|c|}{ Estadísticos descriptivos } \\
\hline Variable & Media & Desviación típica & N. del análisis \\
\hline V1 & 3,33 & 1,213 & 30 \\
\hline V2 & 1,77 & 1,104 & 30 \\
\hline V3 & 3,47 & 1,074 & 30 \\
\hline V4 & 2,73 & 1,048 & 30 \\
\hline V5 & 2,97 & 1,189 & 30 \\
\hline V6 & 1,63 & 1,066 & 30 \\
\hline V7 & 1,73 & 1,048 & 30 \\
\hline V8 & 1,90 & 1,155 & 30 \\
\hline V9 & 4,13 & 0,973 & 30 \\
\hline V10 & 3,30 & 1,088 & 30 \\
\hline V11 & 3,60 & 0,932 & 30 \\
\hline V12 & 3,63 & 1,066 & 30 \\
\hline V13 & 1,83 & 1,147 & 30 \\
\hline V14 & 2,20 & 1,095 & 30 \\
\hline V15 & 3,80 & 1,095 & 30 \\
\hline V16 & 2,40 & 1,133 & 30 \\
\hline V17 & 3,53 & 0,973 & 30 \\
\hline V18 & 2,53 & 1,074 & 30 \\
\hline
\end{tabular}

La prueba de Bartlett con 153 grados de libertad es significativa al 0,2\%. La tabla 6 muestra la varianza total explicada (en \%) y la varianza acumulada por cada una de las posibles componentes del análisis de componentes principales: 
Tabla 6. Varianza total explicada y acumulada por cada nuevo item.

\begin{tabular}{|c|c|c|c|}
\hline \multicolumn{4}{|c|}{ Autovalores iniciales } \\
\hline Variable & Total & \% de la varianza & \% acumulado \\
\hline V1 & 4,018 & 22,325 & 22,325 \\
\hline V2 & 2,417 & 13,428 & 35,753 \\
\hline V3 & 2,064 & 11,469 & 47,222 \\
\hline V4 & 1,388 & 7,710 & 54,932 \\
\hline V5 & 1,302 & 7,234 & 62,166 \\
\hline V6 & 1,196 & 6,647 & 68,813 \\
\hline V7 & 1,142 & 6,344 & 75,157 \\
\hline V8 & 0,973 & 5,408 & 80,565 \\
\hline V9 & 0,811 & 4,508 & 85,073 \\
\hline V10 & 0,629 & 3,496 & 88,569 \\
\hline V11 & 0,541 & 3,004 & 91,573 \\
\hline V12 & 0,375 & 2,086 & 93,659 \\
\hline V13 & 0,358 & 1,991 & 95,650 \\
\hline V14 & 0,241 & 1,340 & 96,990 \\
\hline V15 & 0,231 & 1,281 & 98,271 \\
\hline V16 & 0,163 & 0,908 & 99,179 \\
\hline V17 & 0,088 & 0,489 & 99,669 \\
\hline V18 & 0,060 & 0,331 & 100,000 \\
\hline
\end{tabular}

Teniendo en cuenta los porcentajes de varianza explicada, podríamos agrupar las 18 variables en 4 factores $(54,932 \%$ de varianza explicada), por lo que hemos procedido a llevar a cabo un análisis factorial forzado a 4 factores. 
El gráfico de sedimentación ofrece los autovalores correspondientes a cada una de las variables:

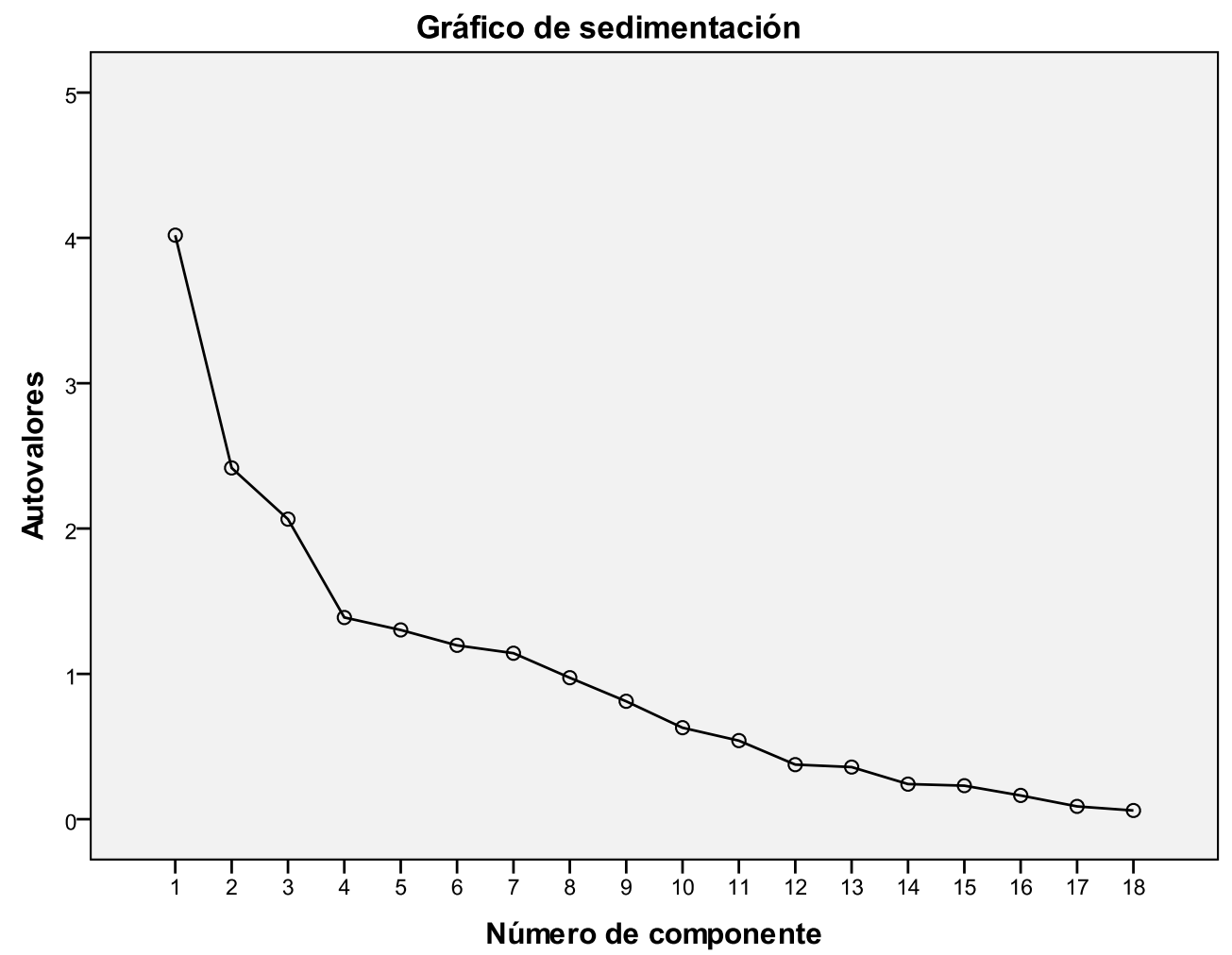

Figura 2. Gráfico de sedimentación de las nuevas variables en estudio. 
La matriz de componentes arroja los siguientes resultados:

Tabla 7. Matriz de componentes.

\begin{tabular}{|c|c|c|c|c|}
\hline \multicolumn{5}{|c|}{ Matriz de componentes } \\
\hline \multirow{2}{*}{ Variables } & \multicolumn{4}{|c|}{ Componente } \\
\cline { 2 - 5 } & $\mathbf{1}$ & $\mathbf{2}$ & $\mathbf{3}$ & $\mathbf{4}$ \\
\hline V1 & 0,570 & 0,148 & $-0,399$ & $-0,251$ \\
\hline V2 & & $-0,312$ & 0,610 & \\
\hline V3 & 0,668 & $-0,218$ & 0,235 & $-0,106$ \\
\hline V4 & 0,261 & & 0,453 & $-0,631$ \\
\hline V5 & 0,625 & $-0,216$ & $-0,348$ & 0,293 \\
\hline V6 & 0,335 & 0,504 & & \\
\hline V7 & 0,346 & & 0,687 & 0,453 \\
\hline V8 & 0,459 & 0,104 & 0,458 & 0,327 \\
\hline V9 & 0,337 & 0,184 & $-0,172$ & 0,620 \\
\hline V10 & 0,777 & $-0,388$ & & \\
\hline V11 & 0,382 & & 0,231 & \\
\hline V12 & 0,681 & & 0,173 & $-0,157$ \\
\hline V13 & 0,500 & 0,546 & & $-0,171$ \\
\hline V14 & 0,585 & 0,221 & $-0,302$ & \\
\hline V15 & 0,150 & 0,767 & & 0,165 \\
\hline V16 & $-0,565$ & 0,462 & 0,247 & \\
\hline V17 & $-0,204$ & 0,652 & 0,413 & $-0,101$ \\
\hline V18 & 0,200 & 0,403 & $-0,131$ & $-0,0145$ \\
\hline
\end{tabular}


Y la matriz de componentes rotados:

Tabla 8. Matriz de componentes rodados.

\begin{tabular}{|c|c|c|c|c|}
\hline \multicolumn{5}{|c|}{ Matriz de componentes rotados } \\
\hline \multirow{2}{*}{ Variable } & \multicolumn{4}{|c|}{ Componente } \\
\cline { 2 - 5 } & $\mathbf{1}$ & $\mathbf{2}$ & $\mathbf{3}$ & $\mathbf{4}$ \\
\hline V1 & 0,526 & 0,520 & $-0,145$ & \\
\hline V2 & & $-0,365$ & 0,480 & 0,332 \\
\hline V3 & 0,557 & & 0,444 & 0,213 \\
\hline V4 & & 0,212 & 0,236 & 0,758 \\
\hline V5 & 0,672 & 0,128 & 0,118 & $-0,404$ \\
\hline V6 & & 0,595 & 0,130 & \\
\hline V7 & & & 0,887 & $-0,102$ \\
\hline V8 & & 0,135 & 0,709 & \\
\hline V9 & 0,135 & 0,235 & 0,271 & $-0,644$ \\
\hline V10 & 0,795 & & 0,339 & 0,115 \\
\hline V11 & 0,228 & & 0,366 & \\
\hline V12 & 0,480 & 0,298 & 0,390 & 0,215 \\
\hline V13 & 0,139 & 0,737 & 0,124 & \\
\hline V14 & 0,435 & 0,526 & & $-0,123$ \\
\hline V15 & $-0,288$ & 0,704 & & $-0,242$ \\
\hline V16 & $-0,773$ & & & \\
\hline V17 & $-0,632$ & 0,381 & 0,223 & 0,229 \\
\hline V18 & & 0,487 & & \\
\hline
\end{tabular}

Por tanto, cada una de las variables anteriores puede agruparse en los siguientes componentes o factores: 
Tabla 9. Agrupación de las nuevas variables por componentes.

\begin{tabular}{|c|c|c|c|}
\hline Factores & Variables & $\bar{x}$ & SD \\
\hline Factor 1 & $\begin{array}{l}\text { V1: Felicitación } \\
\text { V3: Adaptación de las tareas por parte del profesor } \\
\text { V5: Obtención de buenos resultados } \\
\text { V10: Participación } \\
\text { V12: Cobertura de necesidades e intereses } \\
\text { V16: No participación en clase } \\
\text { V17: Descubrimiento de las cosas }\end{array}$ & $\begin{array}{l}3,33 \\
3,47 \\
2,97 \\
3,30 \\
3,63 \\
2,40 \\
3,53\end{array}$ & $\begin{array}{l}1,213 \\
1,074 \\
1,189 \\
1,088 \\
1,066 \\
1,133 \\
0,973\end{array}$ \\
\hline Factor 2 & $\begin{array}{l}\text { V6: Trabajo en equipo } \\
\text { V13: Actuar ante audiencia } \\
\text { V14: Competir } \\
\text { V15: Información sobre contenidos y objetivos } \\
\text { V18: Medios audiovisuales }\end{array}$ & $\begin{array}{l}1,63 \\
1,83 \\
2,20 \\
3,80 \\
2,53\end{array}$ & $\begin{array}{l}1,066 \\
1,147 \\
1,095 \\
1,095 \\
1,074\end{array}$ \\
\hline Factor 3 & $\begin{array}{l}\text { V2: Reproches } \\
\text { V7: Negociación de las decisiones curriculares } \\
\text { V8: Autoevaluación } \\
\text { V11: Clases en inglés }\end{array}$ & $\begin{array}{l}1,77 \\
1,73 \\
1,90 \\
3,60\end{array}$ & $\begin{array}{l}1,104 \\
1,048 \\
1,155 \\
0,932\end{array}$ \\
\hline Factor 4 & $\begin{array}{l}\text { V4: Desafío intelectual } \\
\text { V9: Trabajo individual }\end{array}$ & $\begin{array}{l}2,73 \\
4,13\end{array}$ & $\begin{array}{l}1,048 \\
0,973\end{array}$ \\
\hline
\end{tabular}

Obsérvese que la variable V1 podría encuadrarse tanto en la componente 1 como en la 2, aunque hemos optado por encuadrarla dentro de la componente 1. Las componentes anteriormente obtenidas podrían describirse como sigue:

- Componente 1: Factores motivadores externos más próximos a los internos.

- Componente 2: Factores motivadores externos que realzan la relación con los demás.

- Componente 3: Factores motivadores externos que realzan la participación del alumno.

- Componente 4: Factores motivadores externos que realzan la individualidad del alumno.

Por lo que se refiere al componente 1, podemos observar que la media oscila alrededor de 3,5 sobre 5 lo que nos indica una moderada presencia de los factores motivadores externos 
en el aula, si bien conviene destacar que las variables "obtención de buenos resultados" y, sobre todo, "no participación en clase" se encuentran por debajo del 3. Esto nos indica, por un lado, que los alumnos no consiguen buenos resultados y que sus expectativas de éxito no se cumplen, y por otro lado, que la mayoría de la clase no participa, sino que escucha pasivamente lo que hace y dice el profesor y sus compañeros.

Al observar las medias del componente 2, se puede deducir que la mayoría de los alumnos perciben que los factores motivadores externos no realzan la relación con los demás en clase ya que casi todas están bastante por debajo del 3. Por tanto, no llevan a cabo muchas actividades por parejas o en equipo, no realizan ejercicios que implican competir y el profesor no utiliza demasiado los medios audiovisuales o tecnológicos. Hay una excepción con la variable "información sobre contenidos y objetivos" que se encuentra en una media de 3,8, lo que corrobora que el profesor sí informa a los alumnos con detalle sobre los objetivos y contenidos que se estudian en cada tarea.

Los resultados de las medias del componente 3 muestran que los factores motivadores externos que realzan las participación del alumno son bajos, lo cual muestra que, si bien los alumnos no reciben reproches, ni castigos, lo cual es muy beneficioso para ellos, tampoco participan en la negociación de las decisiones curriculares, es decir, el profesor no da la opción de elegir lo que se estudia en clase, ni se negocia entre profesor y alumnos el tipo de ejercicios que desean realizar. Del mismo modo, tampoco participan en la autoevaluación de sus trabajos. No obstante, hay un dato a resaltar de forma positiva en este componente $\mathrm{y}$ es que el profesor da las clases en inglés.

Finalmente, en cuanto al componente 4, cabe destacar que las medias obtenidas también son llamativas, porque de las dos variables que se estudian en este componente, una de ellas obtiene una media por debajo del tres. Según estos resultados, se consideran insuficientemente los desafíos intelectuales como medio de motivar al alumno, dándose más prioridad al esfuerzo o trabajo individual. En el caso de la otra variable, el resultado de la media está por encima del $4 \mathrm{y}$, al estar relacionada con el hecho de que el alumno trabaje de forma individual, tampoco es muy satisfactorio puesto que va en contra de la interacción con otros compañeros, aspecto muy importante en una clase de lengua extranjera. Este dato coincide con la media obtenida en el componente 2 al abordar el trabajo en equipo, donde los alumnos expresaban que no se realizan demasiadas actividades por parejas o en equipo en estas clases.

Resulta complicado relacionar nuestro estudio empírico con el llevado a cabo por Dörnyei y Csizér (1998:117) debido a la no muy clara correspondencia entre los ítems de la encuesta elaborada por estos autores y los de la encuesta utilizada en nuestro trabajo. No obstante, hemos aproximado ambos cuestionarios y el resultado se refleja en la tabla 10 : 
Tabla 10. Comparación de los mandamientos de Dörnyei y Csizér con las variables estudiadas.

\begin{tabular}{|l|c|}
\hline \multicolumn{1}{|c|}{ Los diez mandamientos de Dörnyei y Csizér } & Variables \\
\hline Dar un ejemplo personal con el propio comportamiento & - \\
Crear una atmósfera relajada y agradable en clase & 14 \\
Presentar las tareas adecuadamente & 15 \\
Desarrollar una buena relación con los estudiantes & - \\
Incrementar la autoconfianza lingǘstica de los estudiantes & $1,3,5$ y 8 \\
Hacer interesantes las clases de lengua & 4 y 18 \\
Promover la autonomía del estudiante & 10 y 17 \\
Personalizar el proceso de aprendizaje & - \\
Incrementar la orientación de los estudiantes hacia los objetivos & 12 \\
Familiarizar a los estudiantes con la cultura de la lengua meta & 11 \\
Incluir el trabajo en grupo de forma regular & 6 \\
Destacar el papel del esfuerzo para llegar al éxito & 9 \\
Utilidad & - \\
Otras recompensas aparte de las notas & - \\
Mantener las reglas que los alumnos aceptaron & 7 \\
\end{tabular}

En la tabla 10 podemos observar el posicionamiento de los ítems de nuestro cuestionario en el modelo de Dörnyei y Csizér. En primer lugar, hemos de subrayar que los ítems 2, 13 y 16 no han podido ser incorporados a la tabla anterior por carecer de un homólogo claro. En segundo lugar, es de destacar que nuestra primera componente "Factores motivadores externos más próximo a los internos" se reparte entre la autoconfianza, la autonomía y la orientación de los estudiantes en el modelo de Dörnyei y Csizér. Adicionalmente, nuestra segunda componente "Factores motivadores externos que realzan la relación con los demás" se corresponde con el trabajo en grupo, la atmósfera relajada entre profesor y alumno, y entre alumnos entre sí, y con una presentación adecuada de las tareas a realizar por los alumnos. Por su parte, nuestra tercera componente "Factores motivadores externos que realzan la participación del alumno" se reparten entre el respeto a las reglas, la autoconfianza y la familiarización con la cultura inglesa. Por último, nuestra cuarta componente "Factores motivadores externos que realzan la individualidad del alumno" se corresponde con el interés y el esfuerzo.

\section{Conclusiones}

A continuación, presentamos nuestras conclusiones en base a los dos objetivos que definimos al comienzo de esta investigación, a saber:

1. El estudio de los factores de la personalidad que están relacionados con los componentes motivacionales en el aula. 
2. El conocimiento de los diferentes niveles de motivación extrínseca en los alumnos de la asignatura de inglés.

Por lo que se refiere al primer objetivo y según hemos señalado anteriormente, podemos concluir que la motivación es igualmente importante para cualquier alumno, independientemente de su factor de personalidad. La mayoría de los alumnos se consideran extrovertidos y les gusta participar en clase, y son menos los que se consideran tímidos. A un porcentaje muy elevado de alumnos, les interesa el idioma extranjero, les gusta y consideran que se esfuerzan, aunque manifiestan querer vivir en España. La mayoría de ellos no se consideran autoritarios y nos les gusta intervenir en los asuntos de los demás. Un gran número de los encuestados se consideran tolerantes y tienen un alto grado de resistencia ante situaciones tales como desorientación, incomprensión, confusión, angustia y ambigüedad.

En cuanto al segundo objetivo de nuestra investigación, podemos decir, a modo de resumen, que los resultados de los alumnos no son exactamente los que ellos esperan y que, por tanto, no se cumplen sus expectativas. Asimismo, los profesores no promueven unas clases muy participativas sino más bien pasivas, y los alumnos no se relacionan mucho entre ellos ya que no se trabaja suficientemente por parejas o en equipo. Un dato positivo es que los alumnos indican que el profesor sí les informa detalladamente acerca de los objetivos y contenidos que se estudian en cada tarea. En la misma línea, también es positivo el hecho de que el profesor da las clases en inglés, pero, sin embargo, no se les da participación en las decisiones curriculares, ni tampoco participan suficientemente en la autoevaluación de su trabajo. De igual manera, la realización de las tareas de clase no supone un desafío intelectual para la mayoría de los alumnos. Finalmente, cabe también destacar como resultado negativo el hecho de que una gran mayoría de los alumnos reconocen que en clase se trabaja de forma individual, evitándoseles así la posibilidad de interacción con sus compañeros, hecho tan importante en una clase de lengua extranjera.

Como conclusión relevante, podemos afirmar que la percepción que los alumnos tienen acerca de su personalidad puede influir de forma positiva en su motivación por aprender una lengua extranjera. No sucede lo mismo con respecto al segundo objetivo, debido a que las respuestas de los alumnos indican que sus niveles de motivación extrínseca ni cumplen sus expectativas ni son útiles para aprender una lengua extranjera.

En este sentido, resaltaremos que cada persona tiene sus rasgos propios por lo que se refiere a la personalidad, y éstos pueden ser positivos o incluso negativos en cuanto al aprendizaje de una lengua extranjera. El rol del profesor puede ser muy importante, ya que gestionará todo lo que ocurra en el aula, y así su actuación con respecto a los alumnos extrovertidos o introvertidos será primordial para reducir o aumentar su ansiedad.

Realmente, lo que los profesores hacen dentro del aula está estrechamente relacionado con lo que piensan y creen al respecto; por ello, sería muy útil potenciar la enseñanza reflexiva (García Sánchez, 2000 y 2001:51-81; Martínez Agudo, 2004:127-144; Quesada Pacheco, 2005:1-19; Hillier, 2005:5-6) puesto que es una forma muy relevante de indagar en la mejora de la eficacia docente. A través de ésta, se le presta atención a lo que significa enseñar, se puede comprender mejor la propia enseñanza y, por lo tanto, se puede mejorar.

Finalmente, consideramos de gran importancia tener en cuenta que, a efectos prácticos y de eficacia en el aula, es conveniente una concepción ecléptica de las teorías de la motivación (Dörnyei, 2008:32-33) abierta a distintas líneas de pensamiento y que tengan en cuenta 
que las aulas no sólo se limitan a las conductas de aprendizaje, sino que son el contexto donde el alumnado desarrolla gran parte de su pensamiento y su comportamiento hacia la vida (sus formas de relacionarse, sus gustos, sus emociones, etc.) y todo ello, por lo tanto, influirá en su motivación y rendimiento a la hora de aprender una lengua extranjera.

En definitiva, se trata de que los alumnos se motiven más para que sean más eficientes al comunicarse en otra lengua y, por consiguiente, conviene prestar atención a todas las competencias que lo permiten (Burillo et al., 2006:9) y a ciertos aspectos psicopedagógicos, como es el caso del aprendizaje significativo, que den sentido y razón a lo que se haga en clase.

\section{Bibliografía}

Barrera Barrera, R. y Reyes Rodríguez, M.C. (2003). "Análisis comparado de las escalas de medición de la calidad de servicio", en Actas de las XIII Jornadas Hispano-Lusas. La Empresa Familiar en un Mundo Globalizado. Lugo: Universidad de Santiago de Compostela, 285-294.

Barrios Espinosa, M.E. (1997). "Motivación en el aula de lengua extranjera", en Encuentro. Revista de Investigación e Innovación en la clase de idiomas, 9: 17-30.

Bernaus, M.; Wilson, A. and Gardner, R.C. (2009). "Teacher's motivation, classroom strategy use, students' motivation and second language achievement", en Porta Linguarum, 12: 25-36.

Burillo, J. et al. (2006). Las lenguas extranjeras en el aula. Reflexiones y propuestas. Caracas: Editorial Laboratorio Educativo.

Dörnyei, Z. (1998). "Motivation in second and foreign language learning", en Lang. Teach. 31: $117-135$.

Dörnyei, Z. (2007). Research Methods in Applied Linguistics. Oxford: Oxford University Press.

Dörnyei, Z. (2008). Estrategias de motivación en el aula de lenguas. Barcelona: Editorial UOC.

Dörnyei, Z. y Csizér, K. (1998). "Ten Commandments for motivating language learners: Results of an empirical study", en Language Teaching Research, 2(3): 203-229.

Ellis, R. (1994). The Study of Second Language Acquisition. Oxford: Oxford University Press.

García Sánchez, M.E. (2000). Las creencias y la actuación del profesor acerca de la motivación en el aula de lengua inglesa. Almería: Servicio de Publicaciones de la Universidad de Almería.

García Sánchez, M.E. (2001). "The English teacher as a reflective practitioner: beliefs on motivation”, en María Elena García Sánchez, (ed.), Present and future trends in TEFL. Almería: Servicio de Publicaciones de la Universidad de Almería, 51-81.

Gómez Martínez, S. (2008). "El aprendizaje del inglés en el ámbito universitario español: perfiles motivacionales e implicaciones en el aula", en Actas del XXXVII Simposio Internacional de la Sociedad Española de Lingüistica (SEL), editadas por Inés Olza Moreno, Manuel Casado Velarde y Ramón González Ruiz, Departamento de Lingüística hispánica y Lenguas modernas. Pamplona: Servicio de Publicaciones de la Universidad de Navarra, 2008. ISBN: 84-8081-053-X. Publicación electrónica en http://www.unav.es/linguis/simposiosel/actas/.

Hair, J.F., Anderson, R.E., Tatham, R.L. y Black, W.C. (2007). Análisis Multivariante. Madrid: Prentice Hall. 
Hillier, Y. (2005). Reflective teaching in further and adult education. Hampshire: Ashford Colour Press.

Liuolienè, A. and Metiūnienė, R. (2006). "Second language learning motivation", en Santalka. Filologija. Edukologija, 14(2): 93-98.

López García, M.D. (2009). ¿Cómo motivar al alumnado para aprender inglés?, en Revista Digital de Innovación y Experiencias Educativas, 25: 1-9.

Madrid, D. (1999). La investigación de los factores motivacionales en el aula de idiomas. Granada: Grupo Editorial Universitario.

Madrid, D. (2001). "Introducción a la investigación en el aula de lengua extranjera", en García Sánchez, M.E. y Salaberri, M.S. (eds.) (2001): Metodología de investigación en el área de filología inglesa. Universidad de Almería: Secretariado de Publicaciones, 11-45.

Marins de Andrade, P.R. (2010). "Estrategias de aprendizaje y desarrollo de la motivación: un estudio empírico con estudiantes de E/LE brasileños", en Porta Linguarum, 14: 141-160.

Martínez Agudo, J. de D. (2004). "Enseñanza reflexiva en el aula de lengua extranjera", en Didáctica (Lengua y Literatura), 16: 127-144.

Pérez Puente, E.M. (2006). Las Webquests como elemento de motivación para los alumnos de educación secundaria obligatoria en la clase de lengua extranjera (inglés). Barcelona: Servicio de Publicaciones de la Universidad de Barcelona.

Quesada Pacheco, A. (2005). "Reflective teaching and its impact on foreign language teaching", en Actualidades Investigativas en Educación, 5: 1-19.

Ramos Nieto, M.C. (2011). "La motivación en la enseñanza del inglés como lengua extranjera", en Aula y Docentes, 32: 39-49.

Uribe, D., Gutiérrez, J. y Madrid, D. (2008). "Las actitudes del alumnado hacia el aprendizaje del inglés como idioma extranjero: estudio de una muestra en el sur de España", en Porta Linguarum, 10: 85-100. 